\title{
Common Pitfalls in Conducting Quantitative Nursing Research: A Commentary and Suggestions to Facilitate Publication of Studies
}

\author{
William Jacobowitz* \\ Adelphi University, College of Nursing and Public Health, Garden City, NY, USA \\ *Corresponding Author - William Jacobowitz, Adelphi University, College of Nursing and Public Health, Garden City, NY, USA. \\ Email: wjacobowitz@adelphi.edu \\ DOI: https://dx.doi.org/10.47988/janany.93687023.1.1
}

\begin{abstract}
Background: This article is a commentary on some of the common pitfalls that many researchers (including nurse researchers) experience when developing and implementing studies which impede their ability to publish their work.

Purpose: The goal is to provide a summary and recommendations to assist novice nurse researchers with respect to some aspects of methodology and statistical analyses that are often overlooked and limit the validity of studies' findings and the reliability of the results.

Discussion: Specific research design attributes, data management and analyses procedures are identified that can be used to improve a study's integrity and appeal to academic journals for publication.

Conclusion: This article attempts to assist nurse researchers of all levels and in a variety of capacities to improve the quality of research and the ability to publish their studies.
\end{abstract}

Keywords: Quantitative, Research, Methodology, Publication

Funding: The author did not receive any funding from the public, commercial, or not-for-profit sectors.

Conflict of Interest: The author declares no actual or potential conflict of interest. 


\section{Common Pitfalls in Conducting Quantitative Nursing Research: A Commentary and Suggestions to Facilitate Publication of Studies}

\section{Introduction}

This article is a commentary about some common quantitative research methodology issues that sometimes impede authors' manuscripts from being published, and to offer a summary and recommendations of ways to avoid those impediments. Quantitative research methodology is a broad and complicated topic. I have attempted to provide information that would be of interest to a wide audience of nurse researchers. For the novice researcher, I offer a basic overview of best practices with respect to research design and the types of statistical analyses that are usually employed. For the somewhat more experienced researcher, I discuss issues of rigor in research and statistics that might provide new insights. In addition, analytical standards change over time. For example, subjects are typically no longer deleted from samples due to missing data as 'a rule of thumb'.

This commentary is only meant to trigger the reader's interest and is not meant to be a complete discussion of any of the topics. Whole books have been written on many of the individual topics touched on in this article. You are referred to standard textbooks on quantitative research methodology and statistical analyses for further details.

Over the years, I have evaluated many research studies as a reviewer for a number of academic journals, a university dissertation advisor, and as a statistician providing analyses for nurse researchers in a variety of clinical and academic settings. As a result, I've observed some common patterns of errors that create difficulty in determining the results and having confidence in the outcomes. Sometimes, these errors result in the need for significant editing of the manuscript which is time-consuming, and in worst-case scenarios they invalidate the results making the manuscript unpublishable. So, it is best to start with a strong research plan. Once you have collected the data, it's nearly impossible to make major changes.

\section{Observations and Recommendations}

What are the most common research design problems that limit a study's ability to be published?

The following is a list and description of 9 common pitfalls.

1. The topic has been thoroughly researched and a new study is not likely to offer new or different findings.

2. The researcher attempts to create a study from previously collected data that was the result of a quality improvement project or poorly designed prior study. Quality improvement projects do not usually possess the rigor of a research study. They are important and necessary mechanisms to identify patient care improvement, but tend to not be designed with sufficient detail to control for a myriad of confounding factors and biases that a reliable and valid research design should contain. Although it is possible to produce a publishable retrospective research study from a quality improvement project, it is limited to those where study-level data has been obtained.
3. A study using previously collected data that has not followed Federal research human subjects protections, i.e., IRB review, data protection guidelines, confidentiality, etc. This is sometimes the result of performing retrospective chart reviews. Even though the patient had signed a consent for treatment, that doesn't automatically permit their clinical data to be used for research purposes unless it is stated in the consent that such might occur. Chart reviews require prior approval from the organization's Institutional Review Board (IRB) which is a Federal requirement of organizations where research is conducted.

4. Failure to obtain permission to conduct the research from the subjects' recruitment site, permission from the subjects (absence of an informed consent for research, or absence of IRB review), and permission from the authors to use the standardized measurement tools. The latter fall under copyright protection and cannot be used without permission unless it is clearly stated by the author in the publication that the tool can be used without the author's permission.

5. Another common mistake is that a researcher believes a certain scenario to be true and then tries to prove it. This generally leads to biased research. Research is an exploratory process. The proper technique is to identify all of the possible predictors of a particular factor of interest, collect data for all or most of them in a valid and reliable manner, and test the relationships using appropriate statistical analyses to identify which are true and which are not. The researcher should be prepared for unexpected results and faithfully report them, and not simply try to prove their original position.

6. The study is under-powered. This usually means that the sample size was too small or the type of data that was collected lacked sufficient precision to be able to identify significant relationships that are inherent in the research design [Type II statistical error] (Plichta \& Kelvin, 2013). This situation may occur when the researcher hasn't performed a sample size estimation prior to data collection or hasn't been able to obtain enough participants to meet the quota of the estimated sample size. In addition, this may occur when the researcher chooses to perform a pilot study due to time or resource constraints. The most efficient way to perform research is to start with a solid design including an adequate sample size for the types of analyses to be conducted.

7. The study only provides descriptive statistics, i.e., means, standard deviations and range of values and doesn't analyze relationships among the variables. Although this type of study can be useful in understanding the prevalence of a situation or issue, it is not very informative with respect to the prediction of that situation. Consequently, this type of manuscript is generally not very competitive related to editors' decisions to publish, unless it is about a very unusual or under-researched topic. 
8. The authors identify significant findings, but do not report the effect sizes. Although this may not affect the ability to get an article published, it does affect the implications for professional practice and for future research which needs to be carefully explained in the article in order to avoid requests for significant editing. The effect size is the magnitude of the relationship among the significant factors. A relationship can be statistically significant, but if the effect size is small, the finding may not have an impact [on practice] (Cohen, 1992; Pek \& Flora, 2018; Warner, 2021).

9. The researchers create their own measurement tools without consideration of reliability and validity measurement, or use tools from published literature which do not have established reliability and validity (absence of psychometric analysis). I recommend using published standardized (reported psychometric statistics) tools whenever possible, except for the recording of the demographic questions which are typically semi-structured, specific to the study's population and developed by the researcher. I will further describe standardized measurement tools and psychometric analysis later in this paper.

\section{How do I choose a topic to research?}

Manuscripts that are most readily published are those that study a topic that will appeal to a large number of RNs in practice or in training, not just those in highly specialized settings or in research environments. Once you've identified a topic, the next step is to find a gap in the current knowledge which will provide an opportunity to contribute new information. Review the current literature and identify whether or not there is a gap. Do not conduct a study in an area that has been highly researched and published, unless there is evidence to refute the preponderance of findings in the literature due to a new piece of evidence, or you intend to conduct a meta-analysis.

\section{Which research design is best with respect to publication?}

There isn't a formula with respect to the best designs regarding publication. However, well-designed studies that have a high degree of validity of results and reliability in their measurement are among the most sought after by journals. The type of design will depend on the nature of the topic, the sample, the amount of time available to conduct the research and the availability of resources to collect data.

Overall, experiments and quasi-experiments are among the best types of healthcare studies with regard to the above. The next-best are correlational designs that control for confounding variables. Experimental designs possess the following characteristics.

a. One or more intervention groups

b. A control group

c. Randomized assignment of subjects to each of the groups

d. Pre and post measurements (longitudinal study) are preferred, if possible

A quasi-experimental design consists of the same characteristics, but lacks randomized assignment of subjects to the groups (Polit \& Beck, 2017).
A type of experiment that is particularly valued for its high level of validity is the cross-over experimental design. This is an experiment in which there are two phases. Phase one is the standard approach with experimental and control groups. In phase two, the control group is exposed to the intervention and thereby becomes the new experimental group. Likewise, the previous experimental group is monitored in the absence of the intervention and becomes the new experimental group. If a significant result is found between the experimental and control groups no matter the timing of the findings (phase 1 or phase 2), it allows for a stronger conclusion of causality between the independent and dependent factors.

A correlational design differs in that it does not possess a control group. It consists of a single group and identifies the significant relationships among the relevant factors by using advanced statistical procedures such as regression analysis (Polit \& Beck, 2012). Of note, the terms independent and dependent variables are reserved for research that employs an experimental design, i.e., possesses an intervention group(s), a control group, and randomized assignment to the groups. Correlational designs utilize the term predictor variable in place of independent, and criterion variable in place of dependent (Salkind, 2010).

\section{Some Data Collection Considerations}

Variables. It is important to identify all of the variables that might be related to the factor of interest (dependent variable), not just the ones that are most readily available. This is because most relationships are multifactorial and are not simple cause and effect situations. It is always necessary to conduct a thorough review of the literature and identify all of the factors that have been observed to be related to your dependent variable. Once you've assembled that list, you'll want to try to collect that data (in addition to the new variable(s) you hypothesize will be related) within your survey. These potentially related variables are identified as control variables and have the potential to create confounding effects within the analyses. A confounder is a variable that is not of central interest in the study, but has an effect on the independent and dependent variables (Meyers et al., 2017; Vander Weele \& Shpitser, 2013). For example, a study of HgbA1c blood levels and a specific diet is likely to be confounded by age and BMI. Confounders are typically controlled through sampling strategies or regression analysis.

Randomization. There are a variety of approaches to randomizing the assignment of subjects to groups in experimental studies. Perhaps the easiest and most reliable is to assign each subject a number and then use a computer program that generates random numbers to select subjects for each of the groups. A readily available program is the RANDBETWEEN function in Microsoft ${ }^{\mathrm{TM}}$ Excel.

Sample selection. Pick a study population for which you are confident to obtain an adequate sample size. Anticipate that $20 \%$ $-30 \%$ of recruits will drop-out or not complete the data collection tool, providing only partial statistics for analyses. Oftentimes the completion rate is dependent on the length of time it takes to complete the survey (number of items or questions).

In terms of estimating the size of the sample that will be needed, the best approach is to use internet-based software. There are many free programs available. A commonly used one is $\mathrm{G}^{*}$ Power 
(Erdfelder et al., 1996). In order to calculate the estimated sample size, you'll need to anticipate the type of statistical test that will be used. You'll also need to enter the desired power, alpha level, effect size and whether you'll employ a one-tailed or two-tailed test. In nursing research, power, alpha level and effect size are typically set at 0.80, 0.05, and medium, respectively (Cohen, 1992). If you are only interested in one type of relationship, whether it be a positive or an inverse relationship, then a onetailed test will maximize the power of your analyses. If you are interested in either type of significant relationship, select a twotailed test.

Generalization. It's important for the reader to be able to generalize the results of your study to their own local populations or population of their interest. Be sure to collect enough demographic information to allow readers to relate the findings to populations other than your sample. As a guide, review articles of studies that have been conducted that are related to your research topic to see which demographic statistics are usually measured.

Standardized measurement tools versus researcherconstructed tools. Except for the demographic questions which are usually developed by the researcher, use standardized measurement tools whenever possible. This will control for various types of biases in the results and increase the readers' confidence in the reliability and validity of your findings. Standardized measurement tools are questionnaires that have been statistically tested to determine their ability to reproduce similar results under similar circumstances (reliability) and their ability to precisely measure the factor of interest (validity). Reliability is generally tested using a Cronbach alpha coefficient. Although there are various opinions, a minimum score of 0.70 is considered a reliable tool (Tavakol \& Dennick, 2011).

Validity is measured in a number of ways. One of the most common is factor analysis. This technique looks for themes in the manner in which the subjects answered the data-gathering instrument in order to determine whether they are consistent with the overall objective of the tool. Each theme is defined as a factor and each factor generally is used to create a subscale within the tool. As long as each subscale has a Cronbach alpha of 0.70 and above, it can be analyzed as a unique variable in addition to the tool's overall score (Note that some scales do not have overall scores and only use the subscale scores for analysis). If the overall score of the tool has been found to be significant with another variable when testing your hypotheses, it is generally appropriate to also test the tool's subscale scores for significance.

Research question. The study should state a research question. It is a brief statement of the reason for your research study using general informal language in the form of a single question. This question is used to formulate the hypotheses.

Hypotheses. These are a series of simple statements indicating the expected relationships to be found among two or more variables. You will use statistical tests to confirm or reject each of the hypotheses in order to draw conclusions and to answer the research question. Most published studies contain 3 to 5 hypotheses.

\section{Data Preparation and Statistical Analysis}

When entering the statistics into a database from paper or computerized surveys, be sure that the scores are properly formatted. Mistakes frequently occur due to researchers not transposing the scores of negatively worded standardized measurement tool items or not performing some of the unique calculations some tools require. It's important to review a tool's scoring guidelines as published by its author to be sure that the results are accurate and can be compared to those of published studies that have used the same tool.

It is often useful to enter the statistics into a database that permits calculations such as Microsoft ${ }^{\mathrm{TM}}$ Excel, as opposed to entering the statistics directly into a statistical software program. Most programs usually have limitations in the way that data can be formatted or calculated. Once data have been entered into the database (such as Excel), you'll want to create several new variables. These are the overall scores and possibly the subscale scores for each of the tools. Within Excel, there is a feature to calculate the sum and the mean (depending on the tool's scoring guidelines) of strings of numbers in specific database cells; allowing for the creation of a column or columns of scores representing the overall (and subscale) scores for each of the tools. Once the statistics have been properly formatted for analysis within the database, it is a simple process to import the data into a statistical software program for analysis.

Determine the completeness of the data. It's not unusual for subjects to occasionally skip over a survey item or prematurely finish without providing responses to all the questions. In the past, incomplete cases (subject data) were simply removed from the analysis (casewise deletion). Current thinking is that removing cases distorts the findings and introduces bias with respect to the findings being representative of the sample. Most experts recommend replacing limited amounts of data whenever possible.

There are three types of missing data: (1) accidental or missing completely at random (MCAR); (2) unexpectedly missing where there are data that explain the missingness, known as missing at random (MAR); and, (3) deliberate omissions on the part of the subject or missing not at random (MNAR). MCAR is generally perceived to be due to human error and is characterized by nonsystemic omissions (randomly missing). Missing data due to MCAR and MAR are generally replaced by the researcher as long as these are $10 \%-20 \%$ of the statistics or less. The method to determine whether or not the missing values are MCAR or MAR is to look for patterns in the omissions. If none exist, you can assume it is MCAR; or if the patterns can be explained by a second variable, you can assume it is MAR. In both circumstances, you should substitute values (Ali et al., 2011).

The substitute data is an estimation of how the subject might have answered the question based on how they answered the other questions within that specific standardized measure. The best method to replace missing values is a procedure called multiple iteration. However, it requires specialized software that is usually unavailable to researchers outside of research institutions due to its cost. Other valid but less regarded procedures involve using linear interpolation which is part of many statistical software programs, or simply inserting the mean of the previous answered 
scores within that tool or subscale. Each method has its strengths and weaknesses (Lang \& Little, 2018).

When a pattern exists with regard to the missing values, i.e., a group of the subjects declined to answer a specific item, or they ceased answering, presumably due to fatigue or confusion about how to answer the question or item, then the missing data is considered missing not at random (MNAR). Since the omissions are the result of intent, data cannot be substituted. However, the subject or case would not be deleted. Instead, the data the subject had provided would be used in the analyses to the extent possible, bearing in mind that a tool's overall score might need to be calculated in an alternative manner since some of the values are missing, e.g., use of the means of the scores (not dependent on the number of answers) instead on the sums of the scores.

Evaluate the Normality of the Data Distributions. Normally distributed data follows established rules of probability resulting in more valid analyses and findings than skewed data (nonnormally distributed). The evaluation of normality is a somewhat subjective process. Usually, normal distributions are bell-shaped when scores are plotted on the X-axis and the frequency of each of those scores on the y-axis. The mean and the median of the distributions are the same or at least very similar. The skewness statistic is typically between +1 and -1 , and the kurtosis statistic is relatively close to 0 . Often a histogram chart of the distribution is generated from the statistical software for examination to confirm a bell-shaped distribution. In addition, the Kolmogorov-Smirnov or Shapiro-Wilks tests may be employed with a p-statistic greater than 0.001 suggesting normality (Meyers et al., 2017). It is essential to evaluate the normality of the distributions of each group within a variable when performing between-group analyses, such as t-Tests and analyses of variance (ANOVA and ANCOVA), and not simply the overall scores for the measurement tool. Normal distributions do not always adhere to the above evaluation techniques and judgement needs to be employed. Most tests perform reasonably well in close-to-normally distributed situations with larger sample sizes (greater than 100). With smaller samples, caution should be exercised.

The determination of normality of the distribution informs the researcher as to the type of test to be performed. With a normally distributed criterion (dependent) variable, you should use a parametric test. Similarly a skewed or kurtotic distribution would require the use of a non-parametric test. When performing correlations or linear regression analysis, the normality of the data can be determined by viewing the normal Q-Q plot of the regression standardized residual. This can be obtained using most statistical software by performing regression analysis of the predictor and criterion variables. The data points in the plot should appear relatively close to the straight line which is the predicted value. Also, a scatter plot chart will be produced at the same time. If the data is normally distributed, the points should be scattered without any pattern (Meyers et al., 2017).

\section{Testing Relationships among Variables}

The first step when testing relationships among variables is to set the alpha level. This is the criterion by which to determine statistical significance. It is usually 0.05 in nursing research and significant results are documented as $\mathrm{p}<0.05$. Next, you'll need to test the criterion (dependent) variable against each of the demographic and other study variables to determine all that are significantly related, using univariate (such as ANOVA) and bivariate (such as Pearson correlation coefficients) tests. This will allow you to determine which variables are significantly related with respect to the variables to be tested in your hypotheses. These related variables, known as co-variates, may be confounders (see section on "Variables") and need to be controlled through the use of specific analytic techniques when testing the hypotheses.

At this stage of the analysis, it is typical to find multiple significant relationships. When co-variates have been identified, it is then necessary to analyze the data using analysis of covariance (ANCOVA) or multiple regression analysis. When performing the latter, the co-variates are entered into the analysis as a group of predictors in the first step of the analysis, and may be labelled control variables. In the following steps, the predictor variables related to the hypotheses being tested are entered. As a result, any of the significant relationships observed among the hypotheses tests will have been adjusted with respect to the influence of the control variables (potential confounders), providing more reliable and valid findings.

There are many free online statistical analysis platforms by which nurse researchers can conduct their own analyses, and a multitude of easy to read user-manuals are available. For some, however, it will be necessary to consult a statistician. It is usually helpful and cost effective to consult with the statistician when designing your study or at least your data collection survey in order to avoid some of the pitfalls that can complicate analyses. Many professional nursing organizations offer small research grants which are often enough to cover statistical analysis costs.

\section{Discussion of Limitations}

Within the discussion section, it is required to disclose the limitations of the study. If it a correlational design, the results cannot be interpreted to mean cause and effect. So, the interpretation of the findings can only assert that there is a relationship among the variables. Only an experimental design can imply causality. Other common limitations are biases in sample selection (representativeness of the population), large amounts of missing data, small effect size, and small sample size. In the latter situation, the most common type of error is one where significant relationships go unidentified. Another common limitation is subject response bias which is when subjects intentionally or unintentionally provide inaccurate information. This is often the result of subject fatigue due to surveys containing many questions and surveys that haven't been standardized resulting in ambiguous, confusing or inadvertently misleading items.

\section{Summary}

In summary, getting your research published can be a challenging task, but you can make the process easier by developing and conducting studies with excellent integrity. The intent of this article is to point out ways in which to develop a research manuscript that is readily publishable. The main focus is on study design, data management and statistical analysis which are generally not the strengths of most researchers. It is the intent 
of the author that researchers of varying skill and experience will find these concepts useful. Finally, the reader is advised to refer to a textbook on clinical research methodology and statistical analysis for further details about the above topics.

\section{References}

Ali, A. M. G., Dawson, S. J., Blows, F. M., Provenzano, E., Ellis, I. O., Baglietto, L., Huntsman, D., Calda, C., \& Pharoah, P. D. (2011). Comparison of methods for handling missing data on immunohistochemical markers in survival analysis of breast cancer. British Journal of Cancer, 104(4), 693-699. https:// dx.doi.org/10.1038/sj.bjc.6606078

Cohen, J. (1992). A power primer. Psychological Bulletin [PsycARTICLES, 112(1). Retrieved from http://www2.psych. ubc.ca/ schaller/528Readings/Cohen1992.pdf

Erdfelder, E., Faul, F., \& Buchner, A. (1996). GPOWER: A general power analysis program. Behavior Research Methods, Instruments, \& Computers, 28(1), 1-11. https://dx.doi. org/10.3758/BF03203630

Lang, K. M., \& Little, T. D. (2018). Principled missing data treatments. Prevention Science, 19(3), 284-294. https://dx.doi. org/10.1007/s11121-016-0644-5
Meyers, L., Gamst, G., \& Guarino, A. J. (2017). Applied multivariate research: Design and interpretation (3rd ed.). Sage Publications, Inc.

Pek, J., \& Flora, D. B. (2018). Reporting effect sizes in original psychological research. Psychological Methods, 23(2), 208225.

Plichta, S. B., \& Kelvin, E. (2013). Munro's statistical methods for health care research (6th ed.). Wolters Kluwer/Lippincott, William \& Wilkins.

Polit, D. F., \& Beck, C. T. (2017). Nursing research: Generating and assessing evidence for nursing practice (10th ed.). Wolters Kluwer/Lippincott, William \& Wilkins.

Salkind, N. (2010). Encyclopedia of research design. https:// dx.doi.org/10.4135/9781412961288 NV - 0

Tavakol, M., \& Dennick, R. (2011). Making sense of Cronbach's alpha. International Journal of Medical Education, 2, 53-55. https://dx.doi.org/10.5116/ijme.4dfb.8dfd

Vander Weele, T. J., \& Shpitser, I. (2013). On the definition of a confounder. Annals of Statistics, 41(1), 196-220. https:// dx.doi.org/10.1214/12-AOS1058

Warner, R. M. (2021). Applied statistics II: Multivariable and multivariate techniques (3rd ed.). Sage Publications, Inc. 